\title{
Reizdarmsyndrom
}

\section{Hilfe über das Darmmikrobiom?}

\author{
Die Therapie des Reizdarms ist eine Crux. Evidenzbasierte Therapieoptionen sind \\ bisher Mangelware. Erfolgversprechend sind jedoch Verfahren, die das Darmmikrobiom \\ beeinflussen wie Probiotika oder die fäkale Mikrobiotatherapie.
}

_ Für die Diagnose Reizdarmsyndrom müssen drei Bedingungen erfüllt sein:

- Die Beschwerden wie Blähungen und Schmerzen müssen länger als 3 Monate anhalten,

- eine andere Erkrankung muss ausgeschlossen sein,

- die Symptome müssen so stark sein, dass dadurch die Lebensqualität relevant beeinträchtigt ist.

Verantwortlich für die Symptomatik sind u.a. viszerale Hypersensitivität, Neuropeptide bzw. Neurotransmitter, eine Aktivierung des Immunsystems, Dysmotilität, eine Störung der Darmbarriere mit gesteigerter intestinaler Permeabilität, zentralnervöse Einflüsse und schließlich das Darmmikrobiom. „Wir wissen heute, dass das Darmmikrobiom ein essenzieller Baustein für die Gesundheit ist und beim Reizdarm eine wichtige Rolle spielt", so Prof. Peter C. Konturek, Saalfeld.

\section{Wie das Mikrobiom das Darmhirn moduliert}

Das intestinale Mikrobiom umfasst mehr als $10^{14}$ Bakterien und besteht aus $>1.000$ verschiedenen Spezies. Beeinflusst wird es durch die Gene des Wirts, Stress, Ernährungsfaktoren, Antibiotika und auch die Art der Geburt. „Kinder, die per Kaiserschnitt auf die Welt kommen, haben ein ganz anderes Mikrobiom als solche, die natürlich geboren werden, und entwickeln später ein erhöhtes Risiko für einen Reizdarm“, so Konturek.

Der Zusammenhang zwischen Darmmikrobiom und verschiedenen Erkrankungen wie Adipositas, Fettleber, Typ-2-Diabetes, Asthma, atopische Dermatitis und Reizdarm ergibt sich daraus, dass das Darmmikrobiom eine Quelle wichtiger Metaboliten darstellt, die auch

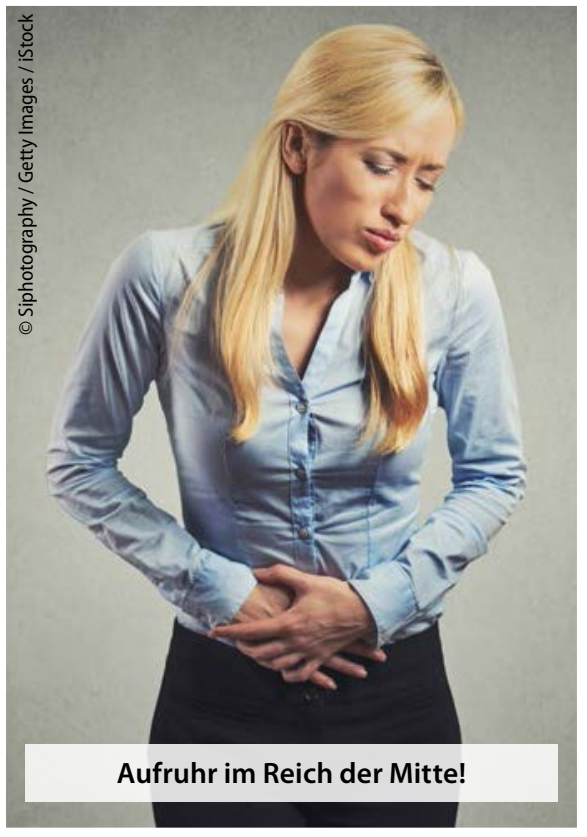

das Darmhirn beeinflussen. Darunter versteht man das 200 Millionen Nervenzellen umfassende autonome enterische Nervensystem, das vitale Darmfunktionen wie Motilität, Sekretion, Absorption, Immunfunktionen und Mikrozirkulation kontrolliert.

\section{Geringere Bakterienvielfalt bei Reizdarmpatienten}

Bei Reizdarmpatienten ist die Anzahl der Bakterienstämme im Darm bzw. deren Vielfalt geringer als bei Gesunden, wobei vor allem weniger Lactobacilli und Bifidobacteriae vorkommen. Auch konnte man die viszerale Hypersensitivität mittels Darmmikrobiom von Reizdarmpatienten auf keimfreie Mäuse übertragen. Diese Beobachtungen begründen die Hoffnung, mit einer Modulation des Darmmikrobioms den Reizdarm erfolgreich behandeln zu können.

\section{Therapeutische Optionen}

In einer Metaanalyse von 43 kontrollierten Studien fanden sich positive Effekte von Probiotika mit einer NNT von 7. Interessanterweise scheint die Wirkung eines bestimmten Probiotikums von der Symptomatik abzuhängen. Bei Blähungen und Schmerz wirken E. coli Nissle und L. acidophilus am besten, bei Obstipation neben E. coli Nissle auch B. animalis und L. casei Shirota, und bei Diarrhö Lactobacilli und Bifidobacteria.

Auch für das schwer resorbierbare Antibiotikum Rifaximin konnte eine effektive Reduktion der Symptome (NNT 10) nachgewiesen werden. Meist ist eine Wiederholung der Therapie erforderlich.

„Wahrscheinlich effektiver als Probiotika ist die fäkale Mikrobiotatherapie mittels Stuhltransplantation“, so Konturek. Bei dieser Therapie wird im Unterschied zu Probiotika eine riesige Kollektion unterschiedlicher Bakterien übertragen, was zum Wiederaufbau des physiologischen Darmmikrobioms führt. Um diese neue Therapieform definitiv beurteilen zu können, sind nun placebokontrollierte Studien erforderlich.

Dr. Peter Stiefelhagen

- 52. Ärztekongress der Bezirksärztekammer Nordwürttemberg, 27.1.2017 in Stuttgart

\section{FAZIT FÜR DIE PRAXIS}

1. Die Störung des Darmmikrobioms spielt eine zentrale Rolle in der $\mathrm{Pa}$ thogenese des Reizdarms.

2. Mit Probiotika können relevante therapeutische Effekte erzielt werden.

3. Eine neue, besonders vielversprechende Therapieoption ist die fäkale Mikrobiotatherapie. 